\title{
Multicast Traffic Engineering for Software-Defined Networks
}

\author{
Liang-Hao Huang ${ }^{*}$, Hsiang-Chun Hsu*, Shan-Hsiang Shen ${ }^{*}$, De-Nian Yang ${ }^{*}$ and Wen-Tsuen Chen ${ }^{*}$ \\ *Institute of Information Science, Academia Sinica, Taipei, Taiwan \\ ${ }^{\dagger}$ Department of Computer Science, National Tsing Hua University, Hsinchu, Taiwan
}

Email: lhhuang@iis.sinica.edu.tw, hchsu0222@gmail.com, \{sshen3, dnyang, chenwt\}@iis.sinica.edu.tw

\begin{abstract}
Although Software-Defined Networking (SDN) enables flexible network resource allocations for traffic engineering, current literature mostly focuses on unicast communications. Compared to traffic engineering for multiple unicast flows, multicast traffic engineering for multiple trees is very challenging not only because minimizing the bandwidth consumption of a single multicast tree by solving the Steiner tree problem is already NP-Hard, but the Steiner tree problem does not consider the link capacity constraint for multicast flows and node capacity constraint to store the forwarding entries in Group Table of OpenFlow. In this paper, therefore, we first study the hardness results of scalable multicast traffic engineering in SDN. We prove that scalable multicast traffic engineering with only the node capacity constraint is NP-Hard and not approximable within $\delta$, which is the number of destinations in the largest multicast group. We then prove that scalable multicast traffic engineering with both the node and link capacity constraints is NP-Hard and not approximable within any ratio. To solve the problem, we design a $\delta$-approximation algorithm, named Multi-Tree Routing and State Assignment Algorithm (MTRSA), for the first case and extend it to the general multicast traffic engineering problem. The simulation and implementation results demonstrate that the solutions obtained by the proposed algorithm outperform the shortest-path trees and Steiner trees. Most importantly, MTRSA is computation-efficient and can be deployed in SDN since it can generate the solution with numerous trees in a short time.
\end{abstract}

Keywords-SDN, multicast, traffic engineering

\section{INTRODUCTION}

Software-defined networking (SDN) provides a new centralized architecture with flexible network resource management to support a huge amount of data transmission [1]. Different from legacy networks, SDN separates the control plane from switches and allows the control plane to be programmable to efficiently optimize the network resources. OpenFlow [1] in SDN includes two major components: controllers (SDN$\mathrm{Cs}$ ) and forwarding elements (SDN-FEs). Controllers are in charge of handling the control plane and install forwarding rules based on different policies, while forwarding elements in switches deliver packets according to the rules specified by the controllers. Compared with the current Internet, routing paths no longer need to be the shortest ones, and the paths can be distributed more flexibly inside the network. It has been demonstrated that SDN provides a better overview of network topologies and enables centralized computation for traffic engineering for multiple unicast flows [2], [3], [4]. However, multicast traffic engineering for multiple multicast trees in SDN has attracted much less attention in previous studies.

Compared to unicast, multicast has been shown in empirical studies to be able to effectively reduce overall bandwidth consumption in backbone networks by around $50 \%$ [5]. It employs a multicast tree, instead of disjoint unicast paths, from the source to all destinations of a multicast group, in order to avoid unnecessary traffic duplication. The current Internet multicast standard, i.e., PIM-SM [6], employs a shortestpath tree to connect the source and destinations, and traffic engineering is difficult for PIM-SM since the path from the source to each destination is the shortest one. A shortest-path tree tends to lose many good opportunities to reduce the bandwidth consumption by sharing more common edges among the paths to different destinations. In contrast, to minimize the bandwidth consumption, a Steiner tree (ST) [7] in Graph Theory minimizes the number of edges in a multicast tree. Nevertheless, ST only focuses on the routing of a multicast tree, instead of jointly optimizing the resource allocations of all trees. Therefore, when the network is heavily loaded, a link will not be able to support a large number of STs that choose the link. Most importantly, Group Table of an SDN-FE will be insufficient to store the forwarding entries of the STs due to the small TCAM size [8].

Compared to the shortest-path routing in unicast, unicast traffic engineering in SDN is more difficult to aggregate multiple flows in Flow Table of an SDN-FE, and the scalability has been regarded as a serious issue in the deployment of SDN for a large network [2], [9]. The scalability problem for multicast communications is even more serious since the number of possible multicast groups is $O\left(2^{n}\right)$, where $n$ is the number of nodes in a network, and the number of possible unicast connections is $O\left(n^{2}\right)$. To remedy this issue, a promising way is to exploit the branch forwarding technique [10], [11], [12], which stores the multicast forwarding entries in only the branch nodes, instead of every node, of a multicast tree, where a branch node in a tree is the node with at least three incident edges. The branch forwarding technique can remedy the multicast scalability problem since packets are forwarded in a unicast tunnel from the logic port of a branch node in SDN-FE [1] to another branch node. In other words, all nodes in the path exploit unicast forwarding in the tunnel and are no longer necessary to maintain a forwarding entry for the multicast group. Furthermore, when a branch node is not multicast capable for a tree (ex. Group Table is full in this paper), local unicast tunneling from a nearby multicast capable node has been proposed in MBONE [13] and PIM$\mathrm{SM}$ to allow multiple unicast tunnels to pass through the branch node to other nodes in the tree (an example will be presented later in this section). Nevertheless, compared to multicast, it is envisaged that local unicast tunneling will incur more bandwidth consumption since duplicated packets will be delivered in a link. Therefore, there is a trade-off between the link capacity and node capacity, because each branch node

\footnotetext{
http://www.cisco.com/c/en/us/td/docs/ios-xml/ios/ipmulti_pim/configuration/xe3s/imc-pim-Xe-3s-book/imc_tunnel.html
} 
can act as either a branch state node with the corresponding multicast forwarding entry stored in Group Table or a branch stateless node that exploits the unicast tunneling strategy.

In comparison with the ST problem, scalable multicast traffic engineering, which jointly allocates the network resources for multiple trees, is much more challenging because both the link capacity and node capacity constraints are involved in the problem. The link capacity constraint states that the total rate of all multicast trees on each link should not exceed the corresponding link capacity, while the node capacity constraint ensures that Group Table of each node is sufficiently large to support the multicast trees with the node as a branch state node. Moreover, scalable multicast traffic engineering with branching forwarding and unicast tunneling techniques is able to allocate the network resources more flexibly. When Group Table of a node is full, unicast tunneling moves the resource requirement from the node to its incident links, whereas the rerouting of the tree is also promising by exploiting the resources of the nearby nodes and links. Therefore, it is necessary for scalable multicast traffic engineering to carefully examine both the routing and the allocation of the branch state nodes of all multicast trees. In this paper, we explore the Scalable Multicast Traffic Engineering (SMTE) problem for SDNs. Given the data rate requirement of each multicast tree, SMTE aims to minimize the total bandwidth cost of all trees, by finding a tree connecting the source and destinations of each group and assigning the branch state nodes for each tree, such that both the link capacity and node capacity constraints can be ensured.

Fig. 1 presents an illustrative example. Fig. 1(a) is the original network with the unit bandwidth cost specified beside each link. The bandwidth cost of each link is the total bandwidth consumption of the link multiplied by the unit bandwidth cost. The node capacity of each node is 1 . The link capacity of edge $e_{s, a}$ is 1 , and the link capacities of the other edges are $\infty$ in this example. There are two multicast trees with both flow rates as 1 . The source of the first tree is $s_{1}=s$, and its destination set is $D_{1}=\left\{d_{1}, d_{2}, \ldots, d_{7}\right\}$. The source of the second tree is $s_{2}=s$, and its destination set is $D_{2}=\left\{d_{1}^{\prime}, d_{2}^{\prime}, \ldots, d_{7}^{\prime}\right\}$. Fig. 1(b) shows the first shortest-path tree (blue) and the second shortest-path tree (red). The branch nodes and branch state nodes of the first tree are $\{c, u, v\}$ and $\{u\}$, respectively. The branch nodes and branch state nodes of the second tree are $\{c, v\}$ and $\{c, v\}$, respectively. Note that $v$ is not assigned as a branch state node of the first tree, and $u$ thus needs to exploit unicast tunneling to $d_{6}$ and $d_{7}$ directly. Therefore, traffic of the first tree are duplicated in edge $e_{u, v}$. On the other hand, if $v$ was assigned as a branch state node for the first tree, traffic duplication in $e_{u, v}$ would be more serious for the second tree since $v$ has three downstream nodes $d_{5}^{\prime}, d_{6}^{\prime}, d_{7}^{\prime}$. The total bandwidth cost of the two shortest-path trees in Fig. 1(b) is 99 .

Afterward, Fig. 1(c) shows the first Steiner tree (blue) and the second Steiner tree (red), and the branch state nodes of the two trees are also $\{u\}$ and $\{c, v\}$, respectively. The total bandwidth cost of the trees in Fig. 11(c) is 103 . Note that the total bandwidth cost in Steiner trees is higher since the assignment of branch state nodes are not carefully examined. Finally, Fig. 1(d) presents the first tree (blue) and the second tree (red) in SMTE with the same branch state nodes specified above. The total bandwidth cost of the trees in Fig. 1(c) is 79 , and here $u$ is directed connected to $d_{1}, d_{2}$, and $d_{3}$ to avoid unicast tunneling, even though the edge cost is higher (i.e., 2) compared to the cost (i.e., 1) of the edge from $v$ in the first Steiner tree. Therefore, this example manifests that it is necessary to consider the tree routing and the assignment of branch state nodes of multiple trees jointly for scalable

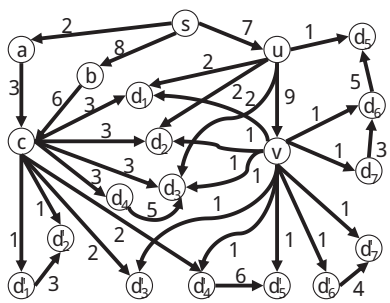

(a) Original network

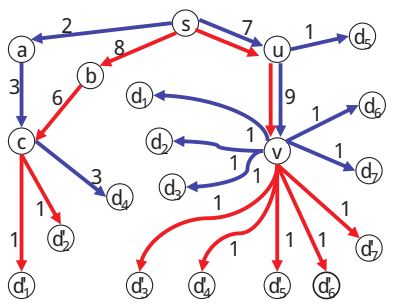

(c) Steiner trees

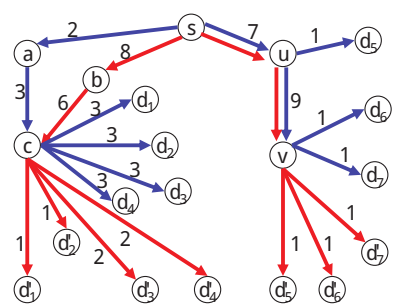

(b) Shortest-path trees

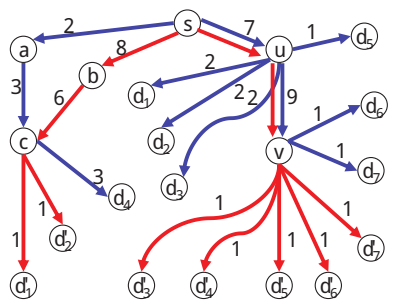

(d) MTRSA
Fig. 1. Comparison of different strategies for multicast traffic engineering

multicast traffic engineering.

SMTE is very challenging. The ST problem is NP-Hard but can be approximated within the ratio $1.55[14]$ and is thus in APX of Complexity Theory. In other words, there exists an approximation algorithm for ST that can find a tree with the total cost at most 1.55 times of the optimal solution. In contrast, we first prove that SMTE-N (i.e., SMTE with only the node capacity constraint, while the link capacity constraint is relaxed) is NP-Hard but cannot be approximated within $\delta$, which denotes the number of destinations of the largest multicast group. Afterward, we prove the SMTE (i.e., with both the link and node capacity) cannot be approximated within any ratio. To solve SMTE-N, we propose a $\delta$-approximation algorithm, named Multi-Tree Routing and State Assignment Algorithm (MTRSA), which can be deployed in SDN-C. MTRSA includes two phases: Multi-Tree Routing Phase and State-Node Assignment Phase, to effectively minimize the total bandwidth cost of all trees according to the node capacity constraint. We first focus on the node capacity (i.e., SMTE-N), instead of the link capacity, because the scalability in Group Table is unique and crucial for SDN and has not been explored in previous studies of multicast tree routing for other networks. Since no $\left(\delta^{1-\epsilon}\right)$-approximation algorithm exists in SMTE$\mathrm{N}$ for arbitrarily small $\epsilon>0$, MTRSA achieves the best approximation ratio. Afterward, we extend MTRSA to support SMTE with the link capacity constraint.

The rest of this paper is organized as follows. Section 2 introduces the related work. Section 3 and 4 formulate SMTE with Integer Programming and describe the hardness results. We present the algorithm design of MTRSA in Section 5, and Section 6 shows the simulation and implementation results on real topologies. Finally, Section 7 concludes this paper.

\section{RELATED WORK}

The issues of traffic engineering for unicast traffic in SDN have attracted a wide spectrum of attention in the literature. Sushant et al. [15] developed private WAN of Google Inc. with the SDN architecture. Qazi et al. [16] designed a new system in SDN to control the middleboxes, and Mckeown et al. [1] studied the performance of OpenFlow in heterogeneous SDN switches. Agarwal et al. [2] presented unicast traffic engineering in an SDN network with only a few SDN-FEs, 
while the other routers in the network followed a standard routing protocol, such as OSPF. However, the above studies focused on only unicast traffic engineering, and multicast traffic engineering for multiple multicast trees in SDN has attracted much less attention.

To support the multicast communications, the current multicast routing standard PIM-SM [6] relies on unicast routing protocols to discover the shortest paths from the source to the destinations for building a shortest-path tree (SPT). However, SPT is not designed to support traffic engineering. Although the Steiner tree (ST) [7] minimizes the tree cost and the volume of traffic in a network, ST is computationally intensive and is not adopted in the current Internet standard. Overlay ST [17], [18], on the other hand, presents an alternative way to construct a bandwidth-efficient multicast tree in the P2P environment. However, the path between any two P2P clients is still a shortest path in Internet, and it is, therefore, difficult to optimize the routing of the P2P tree. Most importantly, both SPT and ST are designed to find the routing of a tree, instead of jointly optimizing the resource allocation of multiple trees.

Flow table scalability is crucial to support large-scale SDN networks due to the limited TCAM size. Kanizo et al. [9], who showed that the major bottleneck in SDN is the restricted table sizes, proposed a framework called Patette to decompose a large SDN table and distribute its entries across a network. Leng et al. [19] proposed a flow table reduction scheme (FTRS) to reduce flow table usage with omnipotent controller functions. DIFANE [8] distributed the flow entries to multiple SDN switches. Zhang at al. [20] built a multicast topology (single backbone tree) for NFV, while Craig et al. adjusted the link weights for shortest-path trees in SDN [21]. Huang et al. also tried to optimize the routing of single multicast tree in SDN [22], [23]. Nevertheless, the above studies were not designed for minimizing the total resource consumption in multicast traffic engineering with multiple trees subject to both the node and link capacity constraints in SDN.

\section{PROBlem Formulation}

In this paper, we explore the Scalable Multicast Traffic Engineering (SMTE) problem for SDN. Given the data rate requirement of each multicast group, SMTE aims to minimize the total bandwidth consumption of all multicast groups in the network, by finding a tree connecting the source and destinations of each group, and assigning the branch state nodes for each tree, such that the number of multicast forwarding states will not exceed the size of Group Table in each node, and the total multicast flows on each edge will not exceed the link capacity. Note that a branch node can only facilitate unicast tunneling for a multicast group if it is not assigned as a branch state node in the corresponding multicast tree.

More specifically, given a network $G(V, E)$, where $V$ and $E$ denote the set of nodes and directed edges, respectively, let $b_{v}$ denote the maximal number of branch state nodes that can be maintained by node 2 . Let $N_{v}^{+}\left(N_{v}^{-}\right)$denote the set of out-neighbor (in-neighbor) nodes of $v$ in $G$. Node $u$ is in $N_{v}^{+}$ $\left(N_{v}^{-}\right)$if $e_{v, u}\left(e_{u, v}\right)$ is a directed edge from $v$ to $u$ (from $u$ to $v$ ) in $E$, and $c_{u, v}$ is the capacity of $e_{u, v}$, while $k_{u, v}$ is the unit bandwidth cost of $e_{u, v}$. Let $\mathcal{T}=\left(T_{1}, T_{2}, \ldots, T_{t}\right)$ denote the set of multicast trees, while $s_{i}$ acts as the root of tree $T_{i} \in \mathcal{T}$, i.e., the source with data rate $f_{i}$, and the destination set $D_{i}$ contains the set of destinations in $T_{i} \in \mathcal{T}$. In the following, we first formally define SMTE, while the derivation of the

\footnotetext{
${ }^{2}$ In the following, we first assume that the memory size allocated in Group Table to maintain the branch state node of each multicast tree is the same, and later we extend it to the general scenario that supports different memory sizes for different multicast trees according to the degrees of the node in the trees [1] in Section $\mathrm{V}-\mathrm{C}$
}

bandwidth consumption will be explained later in this section in the proposed Integer Programming formulation. Dynamic group membership with user join and leave will be discussed later in Section $\mathrm{V}-\mathrm{C}$.

Definition 1. For network $G(V, E)$ and multicast groups $\mathcal{T}$, SMTE is to find the routing of each tree $T_{i}$ in $\mathcal{T}$ spanning $s_{i}$ and $D_{i}$ and assign the branch state nodes in $T_{i}$ to minimize the total bandwidth cost, such that each node $u$ acts as the branch state nodes of at most $b_{u}$ trees, and total multicast bandwidth consumption in each edge $e_{u, v}$ is at most $c_{u, v}$.

In the following, we present the Integer Programming (IP) formulation for SMTE. SMTE includes the following binary decision variables to find the routing of each multicast tree and the assignment of branch state nodes. Let binary variable $\pi_{i, d, u, v}$ denote if edge $e_{u, v}$ is in the path from $s_{i}$ to a destination node $d$ in $D_{i}$ in $T_{i}$. Let integer variable $\varepsilon_{i, u, v}$ denote the number of times that each packet of $T_{i}$ is sent in edge $e_{u, v}$ via multicast (once) or unicast tunneling (multiple times according to the number of tunnels). Let binary variable $\beta_{i, v}$, denote if $v$ is a branch state node in $T_{i}$. Intuitively, when we are able to find the path from $s_{i}$ to each destination node $d$ of $T_{i}$ with $\pi_{i, d, u, v}=1$ on every edge $e_{u, v}$ in the path, together with the set of state branch nodes $\beta_{i, v}$, the routing of the tree (the set of edges $e_{u, v}$ with $\varepsilon_{i, u, v} \geq 1$ ) can be constructed according to the paths from $s_{i}$ to all destination nodes in $D_{i}$.

The objective function of the IP formulation for SMTE is as follows.

$$
\min \sum_{1 \leq i \leq t} \sum_{e_{u, v} \in E} f_{i} \times k_{u, v} \times \varepsilon_{i, u, v} .
$$

The objective function minimizes the total bandwidth cost of all multicast trees. For each tree $T_{i}$, the following constraints first describe the routing assignment (i.e., $\pi_{i, d, u, v}$ ) for the path connecting the source $s_{i}$ and each destination in $D_{i}$. Afterwards, we assign the branch nodes (i.e., $\beta_{i, u}$ ) in different nodes and then derive the bandwidth consumption (i.e., $\varepsilon_{i, u, v}$ ) of $T_{i}$ via multicast and unicast tunneling.

$$
\begin{gathered}
\sum_{v \in N_{s_{i}}^{+}} \pi_{i, d, s_{i}, v}-\sum_{v \in N_{s_{i}}^{-}} \pi_{i, d, v, s_{i}}=1, \forall 1 \leq i \leq t, d \in D_{i}, \\
\sum_{u \in N_{d}^{-}} \pi_{i, d, u, d}-\sum_{u \in N_{d}^{+}} \pi_{i, d, d, u}=1, \forall 1 \leq i \leq t, d \in D_{i}, \\
\sum_{v \in N_{u}^{-}} \pi_{i, d, v, u}=\sum_{v \in N_{u}^{+}} \pi_{i, d, u, v}, \\
\forall 1 \leq i \leq t, d \in D_{i}, u \in V, u \neq d, u \neq s_{i}, \\
\pi_{i, d, u, v} \leq \varepsilon_{i, u, v}, \forall 1 \leq i \leq t, d \in D_{i}, \forall e_{u, v} \in E \\
-\left|D_{i}\right|^{2} \times \beta_{i, u}+\sum_{v \in N_{u}^{+}} \varepsilon_{i, u, v} \leq \sum_{v \in N_{u}^{-}} \varepsilon_{i, v, u} \\
\forall 1 \leq i \leq t, u \in V, u \neq s_{i}, \\
\sum_{1 \leq i \leq t} \beta_{i, u} \leq b_{u}, \forall u \in V \\
\sum_{1 \leq i \leq t} \times \varepsilon_{i, u, v} \leq c_{u, v}, \forall e_{u, v} \in E .
\end{gathered}
$$

The first three constraints, i.e., (1), (2), and (3), are the flow-continuity constraints for each tree $T_{i}$ to find the path from $s_{i}$ to every destination node $d$ in $D_{i}$. More specifically, $s_{i}$ is the source node, and constraint (1) states that the net outgoing flow from $s_{i}$ is one, implying that at least one edge $e_{i, s_{i}, v}$ from $s_{i}$ to any neighbor node $v$ needs to be selected with $\pi_{i, d, s_{i}, v}=1$. Note that here decision variables $\pi_{i, d, s_{i}, v}$ and $\pi_{i, d, v, s_{i}}$ are two different variables because the flow is directed. On the other hand, every destination node $d$ is the flow destination, and constraint (2) ensures that the net incoming flow to $d$ is one, implying that at least one edge 
$e_{i, u, d}$ from any neighbor node $u$ to $d$ must be selected with $\pi_{i, d, u, d}=1$. For every other node $u$, constraint (3) guarantees that $u$ is either located in the path or not. If $u$ is located in the path, both the incoming flow and outgoing flow for $u$ are at least one, indicating that at least one binary variable $\pi_{i, d, v, u}$ is 1 for the incoming flow, and at least one binary variable $\pi_{i, d, u, v}$ is 1 for the outgoing flow. Otherwise, both $\pi_{i, d, v, u}$ and $\pi_{i, d, u, v}$ are 0 . Note that the objective function will ensure that $\pi_{i, d, v, u}=1$ for at most one neighbor node $v$ to achieve the minimum bandwidth consumption. In other words, both the incoming flow and outgoing flow among $u$ and $v$ cannot exceed 1.

Constraints (4) and (5) are formulated to find the routing of the tree and its corresponding branch state nodes, i.e., $\varepsilon_{i, u, v}$ and $\beta_{i, u}$. Constraint (4) states that $\varepsilon_{i, u, v}$ is at least 1 if edge $e_{u, v}$ is included in the path from $s_{i}$ to at least one $d$, i.e., $\pi_{i, d, u, v}=1$. The tree $T_{i}$ is the union of the paths from $s_{i}$ to all destination nodes in $D_{i}$. Constraint (5) is the most crucial one. For each node $u$ in $T_{i}$, if it is not a branch state node, i.e., $\beta_{i, u}=0, u$ does not maintain a forwarding entry of $T_{i}$ in Group Table and thereby facilitates unicast tunneling. In this case, constraint (5) and the objective function guarantee that the number of packets received from an incoming link $e_{v, u}$ must be the summation of the number of packets sent to every outgoing link $e_{u, v}$. By contrast, when $\beta_{i, u}=1$, constraint (5) becomes redundant because the Left-Hand-Side (LHS) is smaller than 0 and thereby imposes no restrict on the Right-Hand-Side (RHS). In this case, constraint (4) ensures that $\varepsilon_{i, v, u}=1$ for every incident edge $e_{v, u}$ with $\pi_{i, d, v, u}$ as 1 . Therefore, $u$ is multicast capable for $T_{i}$, and each packet is delivered once in every incident link.

The last two constraints are capacity constraints. Constraint (6) states that each node $u$ can act as a branch state node of at most $b_{u}$ trees in $\mathcal{T}$, while constraint (7) describes that the total multicast bandwidth consumption of in each directed edge $e_{v, u}$ cannot exceed $c_{u, v}$.

\section{HARDNESS RESULTS}

In the following, we first show that SMTE-N is very challenging in Complexity Theory by proving that it is NPHard and not able to be approximated within $\delta^{c}$ for every $c<1$, where $\delta=\max _{1<i<t}\left|D_{i}\right|$. Afterward, we prove that SMTE cannot be approximated within any ratio.

The Steiner tree problem is a special case of SMTEN. However, SMTE-N is much more challenging than the Steiner tree problem because the Steiner tree problem can be approximated within ratio 1.55 and is thus in APX in Complexity Theory. In contrast, we find out that SMTE is much more difficult to be approximated. The following theorem first proves that SMTE-N cannot be approximated within $\delta^{c}$ for every $c<1$, where $\delta=\max _{1 \leq i \leq t}\left|D_{i}\right|$, by a gap-introducing reduction from the $3 \mathrm{SAT}$ problem.

Theorem 1. For any $\epsilon>0$, there exists no $\left(\delta^{1-\epsilon}\right)$-approximation algorithm for SMTE-N, where $\delta=\max _{1 \leq i \leq t}\left|D_{i}\right|$, assuming $P \neq N P$.

Proof: We prove the theorem with the gap-introducing reduction from the $3 \mathrm{SAT}$ problem.

The 3SAT problem is a simplification of the regular SAT problem. An instance of 3SAT is a conjunctive normal form (CNF) in which each clause contains exactly three variables. The 3SAT problem is to decide, given a Boolean expression $\phi$ in CNF such that each clause contains exactly three variables, whether $\phi$ is satisfiable.

For any instance $\phi$ of the 3SAT problem, we build an instance $G(V, E)$ of SMTE-N with two multicast trees, where the destination sets are $D_{1}$ and $D_{2}$. Let $\mathrm{OPT}(G)$ denote the optimal solution of $G$ for SMTE-N. The goals of the reduction are two-fold. 1) If $\phi$ is satisfiable then $\mathrm{OPT}(G) \leq$ $4 p^{q+1}$. 2) If $\phi$ is not satisfiable then $\operatorname{OPT}(G)>\left(4 p^{q+1}\right) \times$ $\left(\max \left\{\left|D_{1}\right|,\left|D_{2}\right|\right\}\right)^{1-\epsilon}$. In the above two goals, $n$ is the number of Boolean variables in $\phi, m$ is the number of clauses in $\phi$, $p=\max \{m, n\}$ and $q$ is a large number (derived later).

To achieve the above goals, we build the instance of SMTE$\mathrm{N}$ from each instance of the 3SAT problem as follows. Given an instance $\phi$ of 3SAT with $n$ Boolean variables $x_{1}, \ldots, x_{n}$ and $m$ clauses $C_{1}, \ldots, C_{m}$, we construct a directed graph $G(V, E)$ in the following way. 1) The node set $V$ is partitioned into four node sets $\{s\}, U, D_{1}$, and $D_{2}$. 2) $U$ includes $2 n$ nodes $u_{1}, \overline{u_{1}}, u_{2}, \overline{u_{2}}, \ldots, u_{n}, \overline{u_{n}}$ (nodes $u_{i}$ and $\overline{u_{i}}$ correspond to the Boolean variable $x_{i}$ ), and for each $i$ with $1 \leq i \leq n$, there are directed edges $\left(s, u_{i}\right)$ and $\left(s, \overline{u_{i}}\right)$. 3) $D_{1}$ has $m p^{q}$ nodes $d_{j}^{(k)}$, where $1 \leq j \leq m$ and $1 \leq k \leq p^{q}$ (nodes $d_{j}^{(k)}$, $1 \leq k \leq p^{q}$, corresponding to $p^{q}$ copies of the clause $C_{j}$ ), and there exists a directed edge $\left(u_{i}, d_{j}^{(k)}\right)\left(\left(\overline{u_{i}}, d_{j}^{(k)}\right)\right)$ if and only if the variable $x_{i}$ ( $\overline{x_{i}}$, resp.) appears in $C_{j}$. 4) $D_{2}$ contains $n p^{q}$ nodes $w_{i}^{(k)}$, where $1 \leq i \leq n$ and $1 \leq k \leq p^{q}$, and there are directed edges $\left(u_{i}, w_{i}^{(k)}\right)$ and $\left(\overline{u_{i}}, w_{i}^{(\bar{k})}\right)$ for each $i, k$ with $1 \leq i \leq n$ and $1 \leq k \leq p^{q}$. Note that $G$ only has the directed edges described above, $p=\max \{m, n\}$, and $q$ is the smallest integer such that $q \geq\left(3+\log _{p} 4\right) / \epsilon$. Fig. 2 presents an illustrative example of an SMTE-N instance.

The cost of each edge from $s$ to $U$ is set as $p^{q}$, and the cost of the other edges are set to be 1 . The capacity of each node is set as 1 . Let $s$ and $D_{1}$ be the source node and destination set of $T_{1}$ respectively, and let $s$ and $D_{2}$ be the source node and destination set of $T_{2}$ respectively.

If $\phi$ is satisfiable, there is a truth assignment to $x_{i}$ such that $\phi$ is true. Let $A=\left\{u_{i}: x_{i}\right.$ is assigned to be true $\} \cup\left\{\overline{u_{i}}\right.$ : $x_{i}$ is assigned to be false $\}$. Consider the tree $T_{1}$ rooted at $s$ that includes 1) the edges between $s$ and $A$, and 2) the edges between $d_{j}^{(k)}$ and one of its neighbor in $A$ (the existence of its neighbor in $A$ comes from that $\phi$ is satisfiable). Consider the tree $T_{2}$ that includes 1) the edges between $s$ and $U \backslash A, 2$ ) the edges between $U \backslash A$ and $D_{2}$. Then $\left(T_{1}, T_{2}\right)$ is a feasible solution of SMTE-N, and it can act as an upper bound of SMTE-N in $G$. The total edge cost of $T_{1}$ is $n p^{q}+m p^{q}$, and the total edge cost of $T_{2}$ is $n p^{q}+n p^{q}$. Since the node capacity is sufficient, the total bandwidth cost of this feasible solution is $3 n p^{q}+m p^{q} \leq 4 p^{q+1}$. Hence, we have $\mathrm{OPT}(G) \leq 4 p^{q+1}$.

On the other hand, if $\phi$ is not satisfiable, let $\left(T_{1}, T_{2}\right)$ be any feasible solution. For $1 \leq k \leq p^{q}$, let $I_{k}$ be the set consisting of every $i$ with $1 \leq i \leq n$, such that $u_{i}$ and $\overline{u_{i}}$ are adjacent to some nodes in $\left\{d_{j}^{(k)}: 1 \leq j \leq m\right\}$ along the edges in $T_{1}$. Since $\phi$ is not satisfiable, $I_{k}$ is not empty for each $k$ with $1 \leq k \leq p^{q}$. By pigeonhole principle [24], there exists at least one $i^{*}$ with $1 \leq i^{*} \leq n$ such that $i^{*}$ is in at least $\frac{p^{q}}{n}$ sets of $\left\{I_{1}, I_{2}, \ldots, I_{p^{q}}\right\}$. In $\bar{T}_{1}$, therefore, $u_{i^{*}}$ has at least $\frac{p^{q}}{n_{q}} \geq p^{q-1}$ downstream destination nodes, and $\overline{u_{i^{*}}}$ has at least $\frac{p^{q}}{n} \geq p^{q-1}$ downstream destination nodes. On the other hand, in $\bar{T}_{2}, u_{i^{*}}$ and $\overline{u_{i^{*}}}$ need to dominate $p^{q}$ downstream destination nodes of $T_{2}$. If $u_{i^{*}}$ or $\overline{u_{i^{*}}}$ is not a branch state node in $T_{1}$, then the total cost is at least $p^{2 q-1}$. On the other hand, if $u_{i^{*}}$ and $\overline{u_{i^{*}}}$ both are branch state nodes in $T_{1}$, since the capacity of node $u_{i^{*}}$ and $\overline{u_{i^{*}}}$ are 1 , neither $u_{i^{*}}$ nor $\overline{u_{i^{*}}}$ are branch state nodes in $T_{2}$. The total cost is at least $p^{2 q}$.

Therefore, the total cost of the optimal solution is larger than $p^{2 q-1}$, and we have $\operatorname{OPT}(G)>p^{2 q-1}=$ $\left(4 p^{q+1}\right)\left(p^{q-2-\log _{p} 4}\right)=\left(4 p^{q+1}\right)\left(p^{q+1}\right)^{\frac{q-2-\log _{p} 4}{q+1}}=$ 


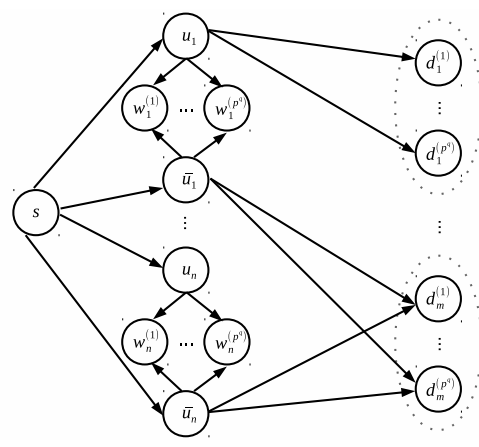

Fig. 2. An illustration of instance building from 3SAT to SMTE-N

$\left(4 p^{q+1}\right)\left(p^{q+1}\right)^{1-\frac{3+\log _{p} 4}{q+1}} \geq \underset{\left(4 p^{q+1}\right)\left(p^{q+1}\right)^{1-\epsilon} \geq}{\geq}\left(4 p^{q+1}\right)\left(\max \left\{\left|D_{1}\right|,\left|D_{2}\right|\right\}\right)^{1-\epsilon}$. Since $\epsilon$ can be arbitrarily $\left(4 p^{q+1}\right)\left(\max \left\{\left|D_{1}\right|,\left|D_{2}\right|\right\}\right)^{1-\epsilon}$. Since $\epsilon$ can be arbitrarily
small, for any $\epsilon>0$, there is no $\left(\max \left\{\left|D_{1}\right|,\left|D_{2}\right|\right\}\right)^{1-\epsilon}$ approximation algorithm for SMTE-N, assuming $\mathrm{P} \neq \mathrm{NP}$. The theorem follows.

In the following, we prove that SMTE cannot be approximated within any ratio.

Theorem 2. For any polynomial time computable function $f$, SMTE cannot be approximated within a factor of $f(|V|)$, unless $P=N P$. In other words, for arbitrary positive integer $k$, SMTE cannot be approximated within $|V|^{k}$.

Proof: Assume, for a contradiction, that there is a polynomial time approximation algorithm $\mathcal{A}$ with the approximation raio $f(|V|)$ for SMTE. This proof will show that $\mathcal{A}$ can be used for deciding the 3 SAT problem in polynomial time, thus implying $\mathrm{P}=\mathrm{NP}$.

Specifically, given a graph $G$, let $\operatorname{OPT}(G)$ denote the optimal solution of $G$ for SMTE. For any instance of the 3SAT problem, we build an instance $G(V, E)$ for SMTE with two multicast trees with the destination sets $D_{1}$ and $D_{2}$. The goals of the reduction are two-fold:

1) if $\phi$ is satisfiable then $\operatorname{OPT}(G) \leq m+3 n$, and

2) if $\phi$ is not satisfiable then $\operatorname{OPT}(\bar{G})>(m+3 n) \times f(|V|)$,

where $n$ is the number of Boolean variables, $m$ is the number of clauses, and $f$ is a polynomial-time computable function.

To achieve the above goals, we build the instance of SMTE from each instance of the 3SAT problem. Given an instance $\phi$ of 3SAT with $n$ Boolean variables $x_{1}, \ldots, x_{n}$ and $m$ clauses $C_{1}, \ldots, C_{m}$, we construct a directed graph $G(V, E)$ such that

1) the node set $V$ is partitioned into four node sets $s, U$, $D_{1}$, and $D_{2}$

2) $U$ contains $2 n$ nodes $u_{1}, \overline{u_{1}}, u_{2}, \overline{u_{2}}, \ldots, u_{n}, \overline{u_{n}}$ (nodes $u_{i}$ and $\overline{u_{i}}$ are corresponding to the Boolean variable $x_{i}$ ), and for each $i$ with $1 \leq i \leq n$, there are directed edges $\left(s, u_{i}\right)$ and $\left(s, \overline{u_{i}}\right)$;

3) $D_{1}$ contains $m$ nodes $d_{1}, \ldots, d_{m}$ (node $d_{j}$ corresponds to the clause $\left.C_{j}\right)$, and there exists a directed edge $\left(u_{i}, d_{j}\right)$ $\left(\left(\overline{u_{i}}, d_{j}\right)\right)$ if and only if the variable $x_{i}\left(\overline{x_{i}}\right.$, resp. $)$ appears in $C_{j}$;

4) $D_{2}$ contains $n$ nodes $d_{1}^{\prime}, \ldots, d_{n}^{\prime}$ and for each $i$ with $1 \leq i \leq n$, there are directed edges $\left(s, d_{i}^{\prime}\right),\left(u_{i}, d_{i}^{\prime}\right)$, and $\left(\overline{u_{i}}, d_{i}^{\prime}\right)$

5) $G$ only has the directed edges described above.

The cost of each edge from $s$ to $D_{2}$ is set as $(m+3 n) \times$ $f(|V|)$, and the cost of every other edge is set to be 1 . The capacity of each directed edge is set to be 1 , and the data rate of each tree is also 1 . The node capacity is set as 2 .
Let $s$ and $D_{1}$ be the source node and destination set of $T_{1}$, respectively Let $s$ and $D_{2}$ be the source node and destination set of $T_{2}$ respectively.

If $\phi$ is satisfiable, there is a truth assignment to $x_{i}$ such that $\phi$ is true, let $W=\left\{u_{i}: x_{i}\right.$ is assigned to be true $\} \cup\left\{\overline{u_{i}}: x_{i}\right.$ is assigned to be false $\}$. Consider the tree $T_{1}$ rooted at $s$ including 1 ) the edges between $s$ and $W$ and 2) the edge between each $d_{j}$ and one of its neighbor in $W$ (the existence of its neighbor in $W$ comes from that $\phi$ is satisfiable). Consider the tree $T_{2}$ which includes 1) the edges between $s$ and $U \backslash W$ and 2) the edges between $U \backslash W$ and $D_{2}$. Then $\left(T_{1}, T_{2}\right)$ is a feasible solution of SMTE and it can act as an upper bound of SMTE in $G$. The total edge cost of $T_{1}$ is $m+n$ and the total edge cost of $T_{2}$ is $2 n$. Since the node capacity is sufficient, the total bandwidth cost of this feasible solution is $m+3 n$. Hence $\mathrm{OPT}(G) \leq m+3 n$.

On the other hand, if $\phi$ is not satisfiable, let $\left(T_{1}, T_{2}\right)$ be any feasible solution. Since $\phi$ is not satisfiable, there is an $i$ such that both edges $\left(s, u_{i}\right)$ and $\left(s, \overline{u_{i}}\right)$ appear in $T_{1}$ for any feasible solution of SMTE, in order to span all destinations in $D_{1}$. Therefore, the edges $\left(s, u_{i}\right)$ and $\left(s, \overline{u_{i}}\right)$ cannot be included in $T_{2}$ due to the link capacity constraint, and $T_{2}$ thereby needs to choose the directed edge $\left(s, d_{i}^{\prime}\right)$. The total edge cost of $T_{2}$ is at least $(m+3 n) \times f(|V|)$ in this case, and the total bandwidth cost of the optimal solution in SMTE is greater than $(m+3 n) \times f(|V|)$. Therefore SMTE cannot be approximated within a factor of $f(|V|)$, unless $\mathrm{P}=\mathrm{NP}$.

\section{Algorithm Design}

In the following, we first propose a $\delta$-approximation algorithm, named Multi-Tree Routing and State Assignment Algorithm (MTRSA), for SMTE-N, where $\delta=\max _{1<i<t}\left|D_{i}\right|$. Note that we first focus on the node capacity, instead of the link capacity, because the scalability in Group Table is crucial in SDN and has not been explored in previous studies of multicast tree routing for other networks. Since Theorem 1 proves that there is no $\left(\delta^{1-\epsilon}\right)$-approximation algorithm of SMTE-N for any $\epsilon>0$, MTRSA achieves the best approximation ratio. Afterward, we extended it to support SMTE.

\section{A. Algorithm Description}

MTRSA includes two phases: 1) Multi-Tree Routing Phase and 2) State-Node Assignment Phase. Multi-Tree Routing Phase first constructs an initial multicast tree for each multicast group to minimize the total bandwidth consumption and balance the distribution of branch nodes in different trees. StateNode Assignment Phase then finds the branch state nodes for each multicast tree to follow the node constraint.

1) Multi-Tree Routing Phase: Initially, Multi-Tree Routing Phase constructs a shortest-path tree with source $s_{i}$ and destination set $D_{i}$ for each tree $T_{i} \in \mathcal{T}$. A node $u$ is full if it acts as a branch node for $b_{u}$ multicast trees. By contrast, $u$ is overloaded if it acts as a branch node for more than $b_{u}$ trees. In this case, $u$ needs to act as a branch stateless node for some of those trees and thereby will incur more bandwidth consumption. To address this issue, after finding the shortest-path trees, if there is an overloaded node, we adjust the local tree routing nearby the overloaded node to move the branch node to another node that has not been full, in order to balance the distribution of branch nodes among different multicast trees.

More specifically, if any node $u$ is an overloaded node and a branch node in any tree $T_{i}$, MTRSA chooses a node $v$ of $T_{i}$ such that: 1) $v$ is a downstream to $u$ in $\left.T_{i}, 2\right) v$ is a branch node or a destination node of $T_{i}$, and 3 ) there is no other branch node or destination node in the path from $u$ to $v$ in $T_{i}$. In other words, $v$ is a nearby downstream branch node of $u$ and 
a destination node. MTRSA reroutes the path (from $u$ to $v$ ) to another path (from $w$ to $v$ ) as follows, in order to alleviate the storage load in $u$. Let $\ell$ denote the total bandwidth cost of the path from $u$ to $v$ in $T_{i}$. We find a new path from $w$ to $v$ such that: 1) the total cost of the new path is at most $\ell, 2$ ) the new path does not pass through any exiting node in $T_{i}$, and 3 ) this new path starts from an on-tree node $w$ such that i) it is not a leaf node of $T_{i}$, and ii) it is not full or overloaded. We update tree $T_{i}$ by substituting the old path from $u$ to $v$ in $T_{i}$ with the new path from $w$ to $v$, and the overload situation in $u$ can be alleviated accordingly. Afterward, we process every other downstream branch node $v$ of $u$ until $u$ is no longer a branch node for $T_{i}$. The above process is repeated for every tree $T_{i^{\prime}}$ iteratively until $u$ is no longer overloaded.

Example. Consider the following example in Fig. 3(a) Let $G(V, E)$ be the network with two multicast trees $T_{1}$ and $T_{2}$ with the data rate as 1 . The number on each edge is the unit bandwidth cost of this edge, and the node capacity of each node is 1 . The source $s_{1}$ of the first tree $T_{1}$ is $s$ with the corresponding destination set $D_{1}=\left\{d_{1}, d_{2}, \ldots, d_{8}\right\}$, while the source $s_{2}$ of $T_{2}$ is also $s$, but the destination set is $D_{2}=$ $\left\{d_{1}^{\prime}, d_{2}^{\prime}, \ldots, d_{6}^{\prime}\right\}$. In Multi-Tree Routing Phase, we first find the blue and red shortest-path trees $T_{1}$ and $T_{2}$ in Fig. 3(b) Afterward, we adjust the multicast trees for overloaded nodes. Specifically, the node capacity of $a$ is 1 , but $a$ is a branch node of both $T_{1}$ and $T_{2}$. Therefore, $a$ is an overloaded node, and MTRSA examines nodes $d_{1}, d_{2}, v, c$, which are downstream nodes of $a$ in $T_{1}$. MTRSA first reroutes the path $\{a, b, c\}$ in tree $T_{1}$. Since node $y$ is overloaded, node $c$ cannot be rerouted from node $y$. In contrast, node $v$ is a full branch node of $T_{1}$, and MTRSA reroutes node $c$ from node $v$ for $T_{1}$ as shown in Fig. 3(c) Note that the bandwidth cost is efficiently reduced since the new path from $v$ to $c$ is much smaller than the one from $a$ to $c$. Therefore, Multi-Tree Routing Phase addresses both the node capacity and the bandwidth consumption for scalable multicast traffic engineering.

2) State-Node Assignment Phase: It is worth noting when the network is heavily loaded, the first phase may not be able to ensure that every overloaded node can be successfully adjusted to balance the distribution of branch nodes in different trees, and State-Node Assignment Phase is crucial in this case to minimize the increment of bandwidth consumption due to unicast tunneling through branch stateless node. More specifically, State-Node Assignment Phase includes two stages: 1) Greedy Assigning Stage, and 2) Local Search Stage. Greedy Assigning Stage assigns the branch state nodes by iteratively maximizing the reduction of the number of branch state nodes, and later in Section $\mathrm{V}-\mathrm{B}$ we prove that the number of branch state nodes reduced by the Greedy Assigning Stage is at least half of the number of branch state nodes reduced by an optimal strategy. Local Search Stage then improves the solution by further alleviating the assignment on overloaded nodes and rerouting the trees to further reduce the total bandwidth cost. We detail the two stages as follows.

For each multicast tree $T_{i}$ obtained in Multi-Tree Routing Phase, let $W_{i}$ denote the set of branch nodes in $T_{i}$, and $W=$ $\cup_{1<i<t} W_{i}$. On the other hand, let $A_{i}$ be the set of branch state nodes in $T_{i}$ to be decided in this phase, and $A_{i}$ thereby is a subset of $W_{i}$. Let $c\left(T_{i}, A_{i}\right)$ denote the total bandwidth cost of $T_{i}$ with the set of branch state nodes as $A_{i}$. More precisely, $c\left(T_{i}, A_{i}\right)=\sum_{v \in A_{i} \cup D_{i}} c\left(P_{v}\right)$, where $P_{v}$ is the path from the closest upstream branch state node in $A_{i}$ or the source to $v$, such that all internal nodes of $P_{v}$ are not in $A_{i}$, and $c\left(P_{v}\right)$ is the cost of all edges in $P_{v}$. In other words, if there is no branch stateless node in $P_{v}$, every packet is delivered only once on every link of $P_{v}$. By contrast, if $P_{v}$ includes a branch stateless node $u$, each packet is sent multiple times on the links from the closest upstream branch state node to $u$, corresponding to the unicast tunneling case.

An assignment $A$ of branch state nodes can be defined as follows: $A$ is a 0,1 -matrix with the rows indexed by $\{1, \ldots, t\}$ and columns indexed by $W$, such that 1) the 1 's in row $i$ can only be the columns indexed in $W_{i}$, and 2) the number of 1 's in column $w \in W$ is no more than the node capacity $b_{w}$. We assign a branch state node $w \in W$ to tree $T_{i}$ if and only if the $(i, w)$ entry of $A$ is 1 . In other words, the first condition ensures that a branch state node can only be assigned to a branch node of $T_{i}$, while the second condition is the node capacity constraint. Given an assignment $A$ of branch state nodes, let $A_{i}=\{w \in W:$ the $(i, w)$ entry of $A$ is 1$\}$ denote the set of branch state nodes for $T_{i}$, and the total bandwidth cost for the set $\mathcal{T}$ of all multicast trees with the state-node assignment $A$ is $c(\mathcal{T}, A)=\sum_{1<i<t} c\left(T_{i}, A_{i}\right)$. Since an assignment $A$ of branch state nodes can also be regarded as a subset of $N$, where $N=$ $\{1, \ldots, t\} \times W$, let $\mathcal{M}$ be the family of subsets of $N$ satisfying the above two conditions (hence $\mathcal{M}$ is the family of all feasible assignments of branch state nodes to $\mathcal{T})$, and we use $c(\mathcal{T}, \varnothing)$ to denote the total bandwidth cost of $\mathcal{T}$ without assigning any branch state node. Now let the set function $z: \mathcal{M} \rightarrow \mathbb{R}$ such that $z(A)$ represents the cost reduced by assignment $A$. More formally, $z(A)=c(\mathcal{T}, \varnothing)-c(\mathcal{T}, A)$ for each $A \in \mathcal{M}$.

The above matrix representation plays a crucial role in Greedy Assigning Stage when we prove the quality of the state-node assignment based on Matroid Theory later in Section $\mathrm{V}-\mathrm{B}$. This stage starts from a branch state node assignment $\varnothing$ and cost $c(\mathcal{T}, \varnothing)$, and iteratively assigns one branch state node for a tree in $\mathcal{T}$ until no more assignment can reduce $c(\mathcal{T}, A)$. More precisely, in each iteration, if the present branch state node assignment is $A \in \mathcal{M}$, we choose an element $x$ in $N-A$ such that: 1) $A \cup\{x\}$ is in $\mathcal{M}$ and follows the node capacity constraint, and 2) $z(A \cup\{x\})=\max _{y \in(N-A)} z(A \cup$ $\{y\})$. In other words, the first condition guarantees that the new assignment is feasible, whereas the second condition chooses the node leading to the maximal reduction on $c(\mathcal{T}, A)$. Afterward, Local Search Stage first adjusts the assignment of branch state nodes for overloaded nodes iteratively. In each iteration, we first extract an overloaded node $u$ and then compute the reduction of the bandwidth cost with a branch state node assigned to $u$ for each tree $T$ spanning $u$, assuming that the state-node assignment of other nodes are not changed. Afterward, this phase sorts the trees according to the bandwidth reduction and chooses the $b_{u}$ trees with the largest reduction, whereas the branch state nodes are assigned to them accordingly. This stage is repeated until all overloaded nodes are carefully examined. Afterward, this stage reroutes the paths from other branch nodes of a tree in order to find a smaller tree with the same assignment of branch state nodes. More specifically, for any branch node $u$ in tree $T_{i}$, we choose nodes $v$ and $w$ of $T_{i}$ in the same way as the Multi-Tree Routing Phase in order to find a new path from $w$ to $v$, and $w$ is not full.

Example. In Greedy Assignment Stage of the State-Node Assignment Phase, when there is no branch state node, the total bandwidth cost in Fig. 3(c) is $c(\mathcal{T}, \varnothing)=c\left(T_{1}, \varnothing\right)+c\left(T_{2}, \varnothing\right)=$ $142+92=234$. If we assign a branch state node on $v$ for tree $T_{1}$, the bandwidth cost of the path $s, a, u, v$ can be reduced by $(4-1)$ times since there are 4 downstream destination nodes $d_{3}, d_{4}, d_{5}, d_{6}$ of $v$ in $T_{1}$. The reduced cost is the largest among all possible branch state node assignments. Therefore, MTRSA first assigns a branch state node on $v$ for tree $T_{1}$ with the cost reduced by $(4-1) \times\left(k_{s, a}+k_{a, u}+k_{u, v}\right)=63$. It then assigns a branch state node on $y$ to $T_{2}$ with the cost reduced by $(3-1) \times\left(k_{s, w}+k_{w, y}\right)=30$. Afterward, node $a$ is assigned as a branch state node for $T_{2}$ with the cost reduced by 


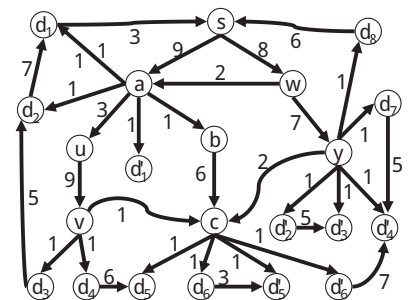

(a) Original network

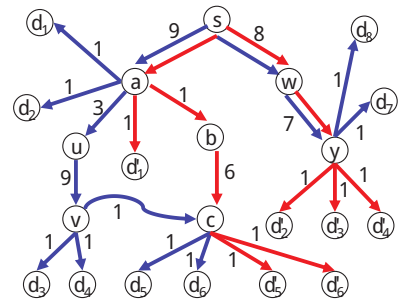

(c) Multi-Tree Routing Phase

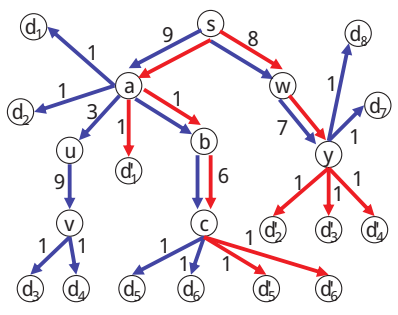

(b) Shortest-path trees

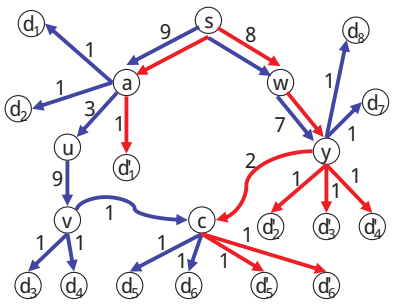

(d) State-Node Assignment Phase
Fig. 3. An example of MTRSA

$(3-1) \times\left(k_{s, a}\right)=18$, and node $c$ is assigned as a branch state node for $T_{2}$ with the cost reduced by $(2-1) \times\left(k_{a, b}+k_{b, c}\right)=7$. In Local Search Stage, there are three overloaded nodes $a, c$, and $y$. For overloaded node $a$, this phase moves the branch state node on $a$ from $T_{2}$ to $T_{1}$ without changing the branch state nodes of the other nodes. If we assign a branch state node on $a$ to $T_{1}$, it becomes possible to reduce the cost of $T_{1}$ by $(3-1) \times\left(k_{s, a}\right)=18$. In contrast, if we assign a branch state node on $a$ to $T_{2}$, we are able to reduce the cost of $T_{2}$ by only $(2-1) \times\left(k_{s, a}\right)=9$. Nodes $c$ and $y$ are then processed similarly. Finally, in Fig. 3(d), since node $y$ has been a branch state node, node $c$ can be re-routed to node $y$ in $T_{2}$, and the total bandwidth consumption is reduced from 107 in Fig. 3(c) to 93 in Fig. 3(d) accordingly.

\section{B. Approximation Ratio and Time Complexity}

In the following, we first examine the quality of assignment for branch state nodes in the second phase. We prove that $(N, \mathcal{M})$ is a matroid and the set function $z: \mathcal{M} \rightarrow \mathbb{R}$ is a nondecreasing submodular set function. Therefore, according to the Matroid Theorem for maximizing submodular set function [25], we have the following theorem.

Theorem 3. The number of branch state nodes reduced by the Greedy Assignment Stage is at least one half of the branch state nodes reduced by the optimal assignment of branch state nodes.

Proof: In the following, we first prove that $\mathcal{M}$ is a matroid. $\mathcal{M}$ is the family of subsets of $N=\{1, \ldots, t\} \times W$ (i.e., we put the elements of $N$ in a $t \times|W|$ array) such that the elements in the $i$-th row are in the columns indexed by $W_{i}$, and the number of elements in the column indexed by $w$ is at most the capacity of $w$. Hence, by definition, we have: 1) $\varnothing \in \mathcal{M}$, 2) If $A \subseteq B \in \mathcal{M}$, then $A \in \mathcal{M}$, and 3) If $A, B \in \mathcal{M}$ with $|A|<|B|$, then there is an element $b \in B$ such that $A \cup\{b\} \in \mathcal{M}$. Therefore $\mathcal{M}$ is a matroid.

Now we prove that the set function $z: \mathcal{M} \subset 2^{N} \rightarrow \mathbb{R}$ is submodular and nondecreasing. Let $A, B \in \mathcal{M}$ with $A \subseteq B$ and $c \in N$ be the element in row $i$ and column $w$ such that $A \cup\{c\}, B \cup\{c\} \in \mathcal{M}$, since $z(A \cup\{c\})-z(A)$ is the cost reduced by assigning a branch state node in node $w$ to tree $T_{i}$ with branch state node assignment $A$, and since $z(B \cup\{c\})-$
$z(B)$ is the cost reduced by assigning a branch state node in node $w$ to tree $T_{i}$ with branch state node assignment $B$, we have $z(A \cup\{c\})-z(A) \geq z(B \cup\{c\})-z(B)$. Hence, $z$ is submodular. Let $A, B \in \overline{\mathcal{M}}$ with $A \subseteq B$, by definition of $z$, we have $z(A) \leq z(B)$, and $z$ thereby is nondecreasing.

Let $Z_{O P T}$ be $\max \{z(A): A \in \mathcal{M}\}$ and $Z_{G}$ be the result from our algorithm. By a result on maximizing submodular set function on matroid [25], we have $\frac{Z_{O P T}-Z_{G}}{Z_{O P T}-z(\varnothing)} \leq \frac{1}{2}$. Hence, $Z_{G} \geq \frac{1}{2} Z_{O P T}$. The theorem follows.

Then, we prove that MTRSA is a $\delta$-approximation algorithm for SMTE-N, where $\delta$ is the maximum size of the destination sets. Since Theorem 1 proves that there is no approximation algorithm with ratio $\delta^{1-\epsilon}$ for any $\epsilon>0$, the following theorem shows that MTRSA achieves the best approximation ratio. In contrast, since SMTE cannot be approximate within any ratio unless $P=N P$, it is impossible to derive an approximation ratio for any algorithm of SMTE, and we thereby evaluate MTRSA for SMTE in Section DI

Theorem 4. MTRSA is a $\delta$-approximation algorithm for SMTE-N, where $\delta=\max _{1 \leq i \leq t}\left|D_{i}\right|$.

Proof: Let the set of multicast trees $\mathcal{T}^{*}=\left(T_{1}^{*}, \ldots, T_{t}^{*}\right)$ with the assignment $A^{*}$ of branch state nodes be the optimal solution to SMTE-N, and $W^{*}=\cup_{i=1}^{t} W_{i}^{*}$ with $W_{i}^{*}$ as the set of branch nodes of $T_{i}^{*}$, whereas $A_{i}^{*}=\left\{w \in W^{*}\right.$ : the $(i, w)$ entry of $A^{*}$ is 1$\}$ be the set of branch state nodes in $T_{i}^{*}$. Therefore, the optimal bandwidth cost is $c\left(\mathcal{T}^{*}, A^{*}\right)=$ $\sum_{i=1}^{t} c\left(T_{i}^{*}, A_{i}^{*}\right)$. For MTRSA, Multi-Tree Routing Phase first constructs the shortest-path trees $\mathcal{T}^{(1)}=\left(T_{1}^{(1)}, \ldots, T_{t}^{(1)}\right)$, and the rerouting procedure of the Multi-Tree Phase outputs the new trees $\mathcal{T}^{(2)}$. MTRSA finally generates the trees $\mathcal{T}^{(3)}$ with the assignment $A^{(3)}$ of branch state nodes. Let $A$ be an assignment such that for each non-overloaded node $u$ within $\mathcal{T}^{(1)}$, MTRSA assigns a branch state node on $u$ to each tree in $\mathcal{T}^{(1)}$ with $u$ as a branch node. According to the State-Note Assignment Phase, we have $A \subseteq A^{(3)}$. On the other hand, in Multi-Tree Routing Phase, MTRSA updates the trees only when the bandwidth cost does not increase, and the branch state nodes can only reduce the cost. Therefore, we have $c\left(\mathcal{T}^{(3)}, A^{(3)}\right) \leq c\left(\mathcal{T}^{(2)}, A^{(3)}\right) \leq c\left(\mathcal{T}^{(2)}, A\right)$. In the rerouting procedure of Multi-Tree Routing Phase, suppose we reroute $\mathcal{T}$ to $\mathcal{T}^{\prime}$. Since each rerouting step does not create any new overloaded node, and the new path in $\mathcal{T}^{\prime}$ ensures that $c\left(\mathcal{T}^{\prime}, A\right) \leq c(\mathcal{T}, A)$. Therefore, $c\left(\mathcal{T}^{(2)}, A\right) \leq c\left(\mathcal{T}^{(1)}, A\right)$ holds by induction, and we have $c\left(\mathcal{T}^{(3)}, A^{(3)}\right) \leq \bar{c}\left(\mathcal{T}^{(2)}, A\right) \leq$ $c\left(\mathcal{T}^{(1)}, A\right) \leq c\left(\mathcal{T}^{(1)}, \varnothing\right)$. Since the path $P_{s_{i}, d}^{(1)}$ from the source $s_{i}$ to node $d$ in $D_{i}$ in tree $T_{i}^{(1)}$ is the shortest path from $s_{i}$ to $d$ in $G$, and $T_{i}^{*}$ has a path from $s_{i}$ to $d$, we have $c\left(P_{s_{i}, d}^{(1)}\right) \leq c\left(T_{i}^{*}\right)$ for every $i$ and every $d \in D_{i}$. Therefore $c\left(\mathcal{T}^{(3)}, A^{(3)}\right) \leq$ $c\left(\mathcal{T}^{(1)}, \varnothing\right)=\sum_{i=1}^{t} c\left(T_{i}^{(1)}, \varnothing\right) \leq \sum_{i=1}^{t} \sum_{d \in D_{i}} c\left(P_{s_{i}, d}^{(1)}\right) \leq$ $\sum_{i=1}^{t} \sum_{d \in D_{i}} c\left(T_{i}^{*}, A_{i}^{*}\right)=\sum_{i=1}^{t}\left|D_{i}\right| \times c\left(T_{i}^{*}, A_{i}^{*}\right) \leq$ $\delta\left(\sum_{i=1}^{t} c\left(T_{i}^{*}, A_{i}^{*}\right)\right)=\delta \times c\left(\mathcal{T}^{*}, A^{*}\right)$. The theorem follows.

Time Complexity. We first find the shortest path between any two nodes in $G$ with Johnson's algorithm in $O\left(|V||E|+|V|^{2} \log |V|\right)$ time as the pre-processing procedure. Multi-Tree Routing Phase constructs the shortest-path tree for each source $s_{i}$ and its corresponding destination set $D_{i}$, and MTRSA compares the distance from a destination node to all other nodes in $O(|V|)$ time. Processing all $d \in D_{i}$ requires $O\left(|V|\left|D_{i}\right|\right)=O(\delta|V|)$ time, and processing all shortest-path trees requires $O(t \delta|V|)$ time. After constructing the shortest- 
path trees, MTRSA reroutes the paths from each overloaded node to some of its downstream nodes. Since there are at most $t \delta$ branches in the tree set, we reroute at most $\delta$ paths for each branch node, and each rerouting requires the comparison of at most $|V|$ distances of paths. Therefore, the above procedure requires $O\left(t \delta^{2}|V| \log |V|\right)$ time, and Routing Phase requires $O\left(t \delta^{2}|V| \log |V|\right)$ time.

Afterward, in Greedy Assigning Stage of State-Node Assignment Phase, there are at most $t|V|$ branch state nodes required to be assigned, and this stage has at most $t|V|$ iterations. In each iteration, we first derive the minimum cost reduced by assigning a branch state node on each node $v$ of every $T_{i}$ in $O(t|V|)$ time, and we update the cost reduced by assigning each new branch state node to the tree $T_{i}$ in $O\left(\left|T_{i}\right||E|\right)=O(|V||E|)$ time. Therefore, this stage requires $O(t|V|(t|V|+|V||E|))=O\left(t|V|^{2}(t+|E|)\right)$ time. Then, Local Search Stage carefully examines the overloaded nodes. In each iteration, we adjust the branch state nodes on each node $u$ in different trees without changing other branch state nodes to find the new bandwidth cost in $O(t|E|)$ time, and this stage takes $O(t|V||E|)$ time because there are at most $|V|$ iterations. Therefore, State-Node Assignment Phase requires $O\left(t|V|^{2}(t+|E|)\right)$ time to allocate the branch state nodes and $O\left(t \delta^{2}|V| \log |V|\right)$ time for rerouting, and MTRSA requires $O\left(t \delta|V|^{2} \log |V|(t+|E|)\right)$ time.

\section{Extension to SMTE}

For SMTE, since the number of times that each packet is delivered in a link cannot be acquired before assigning the branch state nodes, we first present the concept of weak edge capacity constraint. Let $\varepsilon_{i, u, v}^{\prime}=1$ if $\varepsilon_{i, u, v}$ is a positive integer in our Integer Programming formulation, and $\varepsilon_{i, u, v}^{\prime}=0$ otherwise. MTRSA needs to ensure $\sum_{1<i<t} f_{i} \times \varepsilon_{i, u, v}^{\prime} \leq c_{u, v}$ holds for $\forall e_{u, v} \in E$ before assigning the branch state nodes. In addition, in the general case of SMTE, the storage size of each branch state node $u$ in Group Table is proportional to the degree of $u$ in the corresponding multicast tree [1]. Therefore, let $\beta_{i, u}$ denote the node weight (i.e., storage size) of assigning a branch state node on $u$ to tree $T_{i}$, and $b_{u}$ here denotes the size of Group Table in $u$. For SMTE, we extend MTRSA as follows.

Before Multi-Tree Routing Phase, we sort the multicast trees in $\mathcal{T}$ according to their data rates in the ascending order, $f_{1} \leq f_{2} \leq \cdots \leq f_{t}$. In Multi-Tree Routing Phase, we find the first shortest-path tree $T_{1}$ in $\mathcal{T}$ and decrease the link capacity $c_{u, v}$ for every edge in $T_{1}$ by its flow rate $f_{1}$. Note that any edge $e_{u, v}$ with insufficient residual capacity to support $f_{2}$ will be removed since it cannot support the rest of the multicast flows. The above procedure is repeated for other trees in $\mathcal{T}$.

In the rerouting procedure of Multi-Tree Routing Phase, we reroute each multicast tree $T_{i}$ according to the weak edge capacity constraint, such that any new path from $w$ to $v$ in Section $\mathrm{V}-\mathrm{A}$ must have sufficient capacity to support $f_{i}$. In Greedy Assignment Stage, we find an element $x=(i, u)$ in $N-A$ according to $\frac{z(A \cup\{(i, u)\})-z(A)}{\beta_{i, u}}=\max \left\{\frac{z\left(A \cup\left\{\left(i^{\prime}, u^{\prime}\right)\right\}\right)-z(A)}{\beta_{i^{\prime}}}\right.$ : $\left.A \cup\left\{\left(i^{\prime}, u^{\prime}\right)\right\} \in \mathcal{M}\right\}$, which represents the normalized cost reduction. In other words, the node weight $\beta_{i, u}$ is considered during the assignment of branch state nodes.

In Local Search Stage, optimizing the state-node assignment of one node becomes the same as the knapsack problem because each candidate branch state node now has a profit (i.e., cost reduction) and a size (i.e., node weight), and we exploit Polynomial-Time Approximation Scheme for knapsack [26] to find the solution. In the rerouting procedure of Local Search $\overline{\text { Algorithm } 1 \text { Multi-Tree Routing and State Assignment Algo- }}$ rithm (MTRSA)

Require: A network $G=(V, E)$, source nodes $s_{1}, s_{2}, \ldots, s_{t}$, destination sets $D_{1}, D_{2}, \cdots, D_{t}$, and State-Node table $A$.

Ensure: Multicast trees $T_{1}, T_{2}, \cdots, T_{t}, s_{i}$ is the root of $T_{i}$, and $D_{i}$ is in $T_{i}$.

1: //Multi-Tree Routing Phase

2: for $i \in\{1,2, \ldots, t\}$ do

3: $\quad T_{i} \leftarrow$ shortest path tree containing $D_{i}$ with root $s_{i}$

4

5: for overloaded node $u$ do

6: Reroute an appropriate downstream node $v$ of $u$ to balance the distribution of branch nodes

7:

8: //State-Node Assignment Phase 1) Greedy Assigning Stage

9: $A \leftarrow[\mathbf{0}]$

10: while there is $x \in N-A$ such that $A \cup\{x\} \in \mathcal{M}$ do

11: $\quad x_{\max } \leftarrow \arg \max _{x \in N-A}\{Z(A \cup\{x\}): A \cup\{x\} \in \mathcal{M}\}$

12: $\quad A \leftarrow A \cup\left\{x_{\max }\right\}$

13:

14: //State-Node Assignment Phase 2) Local Search Stage

15: for overloaded node $u$ do

16: Re-assign node state on $u$ to maximizing reduction

17:

18: for overloaded node $u$ do

19: $\quad$ while node $u$ is overloaded do

20: $\quad$ for each node $z$ in $V$ do

21: $\quad$ if node $z$ is not overloaded then

22: $\quad$ Reroute node the downstream node $v$ of $u$ to node $z$

23: $\quad$ Break the for loop

Stage, since now the branch state nodes have been specified, we reroute each multicast tree $T_{i}$ according to the original edge capacity constraint (7), such that any new path from $w$ to $v$ must have sufficient capacity to support $f_{i}$. If the amount of multicast flows in any edge exceeds the capacity constraint, we also reroute its closest upstream state node $u$ in a tree $T_{i}$ to $w$, such that the new path from $w$ to $v$ follows the link capacity constraint.

MTRSA can support the dynamic multicast group membership as follows. When a user $v$ joins or leaves a multicast group, MTRSA adds or trims (if no other users are located downstream to the user) the corresponding branch from the upstream branch node $u$ in the same way as Multi-Tree Routing Phase. Afterward, State-Node Assignment Phase adjusts the new branch state node if necessary. Therefore, it does not need to re-compute the whole tree.

\section{Pseudo Code}

The pseudo code of MTRSA is shown in Algorithm 1

\section{PERFORMANCE EVALUATION}

In this section, we first compare MTRSA and other approaches with real topologies. Afterward, we deploy our algorithm in a small experimental SDN network with HP SDN switches to evaluate the video performance with YouTube HD traffic that requires a large amount of bandwidth consumption..

\section{A. Simulation Setup}

We simulate our algorithm in two real networks: VtlWavenet2011 and Columbus [27]. VtlWavenet2011 includes 91 nodes and 96 links, while Columbus has 70 nodes and 85 


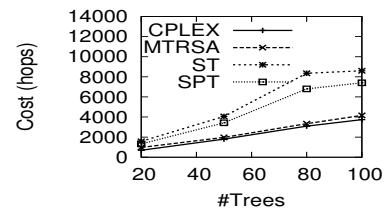

(a) VtlWavenet 2011

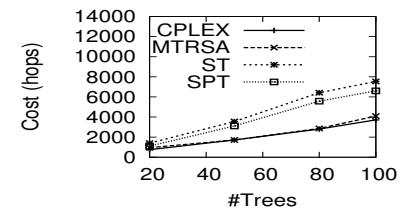

(b) Columbus

Fig. 4. Cost with different $|T|\left(b_{u}=7,|D|=6\right)$

links. Our simulation is divided into small-scale and largescale cases. The numbers of trees in the small-scale cases are smaller than 100, whereas there are more than 2000 trees in the large-scale cases. The link capacity in the topologies is set to the level that the maximal bottleneck link utilization reaches $100 \%$ [28], and the edge cost of each link is set as 1 . We vary the number of multicast trees, the number of destinations, and node capacity. The source and destinations are chosen randomly from each network.

We compare MTRSA with the following algorithms: 1) the shortest-path tree algorithm (SPT), 2) the Steiner tree (ST) algorithm[ [7], and 3) CPLEX [29], which finds the optimal solutions of SMTE problem by solving the IP formulation in Section [III In SPT and ST, the branch state nodes of different trees are randomly assigned to a branch node when node is fully utilized, i.e., the number of branch state nodes reaches the node capacity. Each SPT and ST is added to the network iteratively. If an edge does not have sufficient residual capacity to support the multicast flow of a new SPT or ST, it will be removed accordingly to avoid choosing the edge in the SPT or ST. We implement all algorithms in an HP DL580 server with four Intel Xeon E7-4870 $2.4 \mathrm{GHz}$ CPUs and 128 GB RAM. Each simulation result is averaged over 100 samples.

\section{B. Small-Scale Evaluation}

In the small-scale cases, we compare the total bandwidth costs of all trees in MTRSA, SPT, ST, and CPLEX with different number of trees $(|T|)$, different node capacity $\left(b_{u}\right)$, and different number of destinations $|D|$. As shown in Fig. 4 and Fig. 5] MTRSA generates a solution with the total bandwidth cost very close to the optimal solution. Although SPT chooses the shortest path to the destinations, it does not carefully examine the node capacity, and its cost is thus higher than MTRSA. Compared with SPT, the distance from the source to a destination in ST is usually higher because the path needs to be deviated from the shortest one in order to share more edges with another path. Therefore, more branch nodes are usually involved in ST. Without a sophisticated allocation of branch state nodes, ST incurs a slightly higher cost than SPT due to the additional bandwidth consumption in unicast tunneling for the branch nodes with full Group Table. The difference becomes more significant when the number of trees increases as shown in Fig. 4 . Similarly, Fig. 5 manifests that the total bandwidth cost of each tree increases as the number of destinations grows, because each tree becomes larger in this case.

\section{Large-Scale Evaluation}

In the following, we evaluate MTRSA, ST, and SPT in larger-scale cases, where the number of multicast tree is ranged from 2000 to 10000 , the number of destinations is from 5 to 25 , and the node capacity is between 50 and 250 . Compared with smaller-scale cases, the advantage of MTRSA

\footnotetext{
${ }^{3}$ There are some single-tree multicast routing algorithms with differernt purpuses (such as QoS), but they are not included in this study because ST (i.e., the optimal solution for single tree) outperforms those approaches in terms of the bandwidth consumption.
}

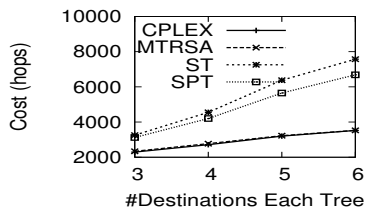

(a) VtlWavenet2011

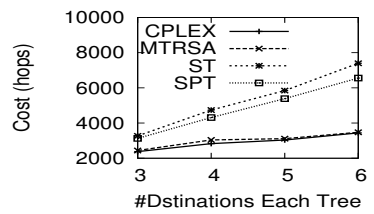

(b) Columbus
Fig. 5. Cost with different $|D|\left(|T|=100, b_{u}=7\right)$

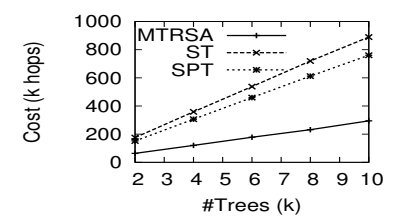

(a) VtlWavenet2011

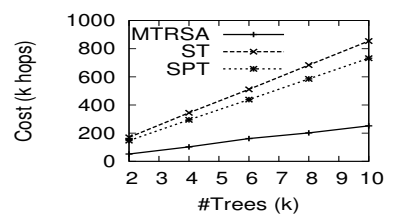

(b) Columbus
Fig. 6. Cost with different $|T|\left(b_{u}=300,|D|=10\right)$

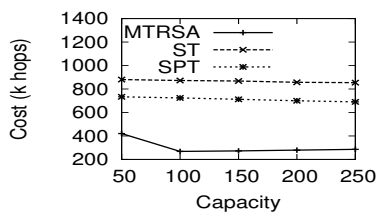

(a) VtlWavenet2011

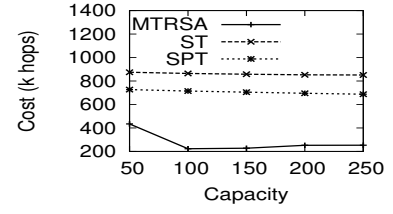

(b) Columbus
Fig. 7. Cost with different $b_{u}(|T|=6000,|D|=10)$

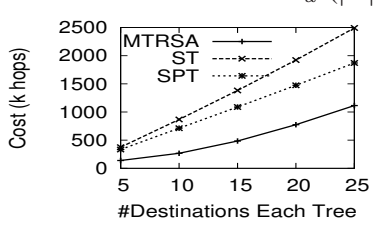

(a) VtlWavenet2011

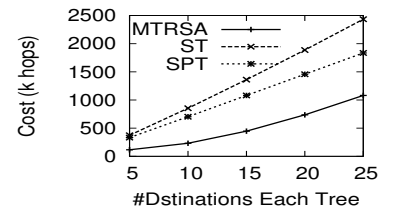

(b) Columbus
Fig. 8. Cost with different $|D|\left(|T|=6000, b_{u}=300\right)$

TABLE I. RUNNING TIME OF MTRSA (SECONDS)

\begin{tabular}{|c|c|c|c|c|c|c|}
\hline$|T|$ & $D \mid=5$ & $D \mid=10$ & $D=15$ & $D=20$ & $|D|=25$ \\
\hline 2000 & 1.00 & 2.29 & 3.01 & 3.43 & 3.99 \\
4000 & 3.09 & 6.33 & 8.71 & 11.06 & 12.39 \\
6000 & 5.82 & 12.52 & 17.94 & 22.37 & 25.53 \\
8000 & 8.80 & 20.10 & 29.89 & 37.52 & 43.78 \\
10000 & 12.83 & 32.32 & 48.44 & 57.01 & 73.40 \\
\hline
\end{tabular}

is more significant in larger-scale cases. In Fig. 6 the total cost increases with the number of trees. For a larger network, the source and any destination are inclined to be located with distantly, but there is also a higher chance to find a node with sufficient capacity as a branch node for rerouting. Therefore, MTRSA effectively reduces the total bandwidth cost by $66 \%$ and 59\%, respectively, compared to ST and SPT. In addition, Fig. 7 shows that the bandwidth costs can be effectively reduced when we increase node capacity, and setting the node capacity as 100 is sufficient for MTRSA. On the other hand, the bandwidth cost grows with the number of destinations, because all trees are required to span more nodes as shown in Fig. 8

Table I summarizes the running time of MTRSA with different $|T|$ and $|D|$. With a smaller input, such as 2000 trees and 5 destinations in each tree, the running time for MTRSA is around 1 second. As $|T|$ and $|D|$ increase, MTRSA only requires around 73 seconds in the largest case with 10000 multicast trees. Therefore, it is envisaged that our algorithm is practical to be deployed in SDN. 
(a) Topology

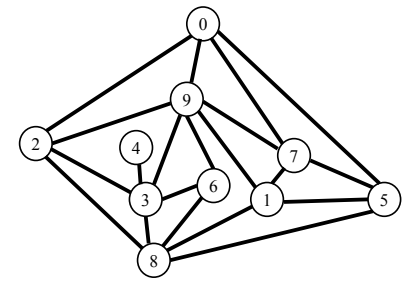

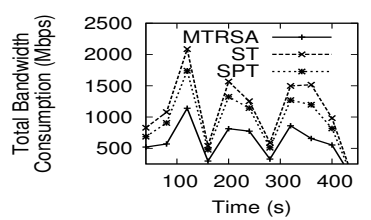

(b) Bandwidth consumption
Fig. 9. Implementation results of the experimental SDN

\section{Implementation}

To evaluate MTRSA in real environments, we implement it in an experimental SDN with HP Procurve 5406zl OpenFlowenabled switches. We use Floodlight as the OpenFlow controller to install the multicast forwarding rules in SDN-FEs. We install multicast group information in group table and create virtual ports mapping to multiple physical ports to forward multicast traffic. MTRSA is running on the top of Floodlight. Our testbed includes 12 nodes and 24 links as shown in Fig. 9 where the link capacity and node capacity are set as $50 \mathrm{Mbps}$ and 5, respectively. We randomly select 10 nodes as the video multicast sources, where each source is connected to a Youtube proxy to facilitate YouTube multicast. We implement the Youtube proxy by using VLC player, which can request video stream from Youtube and work as a video server. To support multicast, we modify TCP to aggregate TCP ACKs from multiple clients. The full-HD test video is in 460 seconds encoded in H.264 with the average bit rate as 10Mbps. For each source, we randomly assign 10 destinations that play videos using the VLC player. Fig. 9(b) shows that the total bandwidth consumption during playback, and we average the bandwidth consumption every 40 seconds. The results manifest that the bandwidth consumption of MTRSA is $46 \%$ and $35 \%$ lower than ST and SPT, respectively. Therefore, MTRSA can effectively support multicast traffic engineering in SDN.

\section{CONCLUSION}

Recent studies on traffic engineering for SDN mostly focus on unicast, while most existing multicast routing algorithms are designed to find the routing of a multicast tree, instead of multiple trees. In this paper, therefore, we have formulated Scalable Multicast Traffic Engineering Problem (SMTE) to minimize the total bandwidth cost according to the link and node capacity constraints for multiple trees in SDN. We have proved that SMTE-N is NP-hard and not able to be approximated within $\delta$, while SMTE cannot be approximated within any ratio. To solve the problem, we have proposed Multi-Tree Routing and State Assignment Algorithm (MTRSA), which is a $\delta$-approximation algorithm for SMTE-N, while MTRSA has been extended to support SMTE as well. Simulation based on real topologies and implementation with Youtube traffic manifest that MTRSA can effectively find the routing of multiple multicast trees and assign the branch state nodes in order to reduce the total bandwidth cost, while the computation time to construct numerous trees is also reasonable for practical SDN. Since the tree obtained by MTRSA is not delay bounded, we will extend it to support QoS multicast in the future work.

\section{ACKNOWLEDGMENT}

This work is supported by MOST 104-2622-8-009-001.

\section{REFERENCES}

[1] McKeown et al., "OpenFlow: Enabling innovation in campus networks," SIGCOMM Comput. Commun. Rev., vol. 38, no. 2, pp. 69-74, Mar. 2008
[2] S. Agarwal, M. Kodialam, and T. Lakshman, "Traffic engineering in software defined networks," in IEEE INFOCOM'13, pp. 2211-2219.

[3] Lazaris et al., "Tango: Simplifying sdn control with automatic switch property inference, abstraction, and optimization," in ACM CoNEXT '14, pp. 199-212.

[4] X. N. Nguyen, D. Saucez, C. Barakat, and T. Turletti, "Optimizing rules placement in openflow networks: Trading routing for better efficiency," in ACM HotSDN '14, pp. 127-132.

[5] R. Malli, X. Zhang, and C. Qiao, "Benefit of multicasting in all-optical networks," Proceedings of the SPIE, vol. 3531, pp. 209-220, Nov. 1998.

[6] Fenner et al., "Protocol independent multicast - sparse mode (pim-sm)," IETF RFC 4601, Dec. 2007.

[7] F. K. Hwang and D. S. Richards, "Steiner tree problems," IEEE Networks, vol. 22, no. 1, pp. 55-89, Oct. 1992.

[8] M. Yu, J. Rexford, M. J. Freedman, and J. Wang, "Scalable flow-based networking with DIFANE," in ACM SIGCOMM '10, pp. 351-362.

[9] Y. Kanizo, D. Hay, and I. Keslassy, "Palette: Distributing tables in software-defined networks," in IEEE INFOCOM'13, pp. 545-549.

[10] D.-N. Yang and W.-J. Liao, "Optimal state allocation for multicast communications with explicit multicast forwarding," IEEE Trans. on Parallel and Distributed Systems, vol. 19, no. 4, pp. 476-488, 2008.

[11] D.-N. Yang and W. Liao, "Protocol design for scalable and adaptive multicast for group communications," in IEEE ICNP'08, pp. 33-42.

[12] I. Stoica, T. Ng, and H. Zhang, "REUNITE: a recursive unicast approach to multicast," in IEEE INFOCOM'00, pp. 1644-1653.

[13] K. Almeroth, "The evolution of multicast: from the mbone to interdomain multicast to internet2 deployment," IEEE Networks, vol. 14, no. 1, pp. 10-20, Jan 2000.

[14] G. Robins and A. Zelikovsky, "Improved steiner tree approximation in graphs," in ACM SODA'00, pp. 770-779.

[15] Sushant Jain et al., "B4: Experience with a globally-deployed software defined WAN," in ACM SIGCOMM'13, pp. 3-14.

[16] Qazi et al., "SIMPLE-fying middlebox policy enforcement using SDN," in ACM SIGCOMM'13, pp. 27-38.

[17] D.-N. Yang and W. Liao, "On bandwidth-efficient overlay multicast," IEEE Trans. on Parallel and Distributed Systems, vol. 18, no. 11, pp. 1503-1515, Nov. 2007.

[18] E. Aharoni and R. Cohen, "Restricted dynamic steiner trees for scalable multicast in datagram networks," IEEE/ACM Trans. on Networking, vol. 6, no. 3, pp. 286-297, Jun. 1998.

[19] B. Leng, L. Huang, X. Wang, H. Xu, and Y. Zhang, "A mechanism for reducing flow tables in software defined network," in IEEE ICC'15, pp. 6924-6929.

[20] S. Q. Zhang, Q. Zhang, H. Bannazadeh, and A. Leon-Garcia, "Network function virtualization enabled multicast routing on SDN," in IEEE ICC'15, pp. 7217-7223.

[21] A. Craig, B. Nandy, I. Lambadaris, and P. Ashwood-Smith, "Load balancing for multicast traffic in SDN using real-time link cost modification," in IEEE ICC'15, pp. 7418-7424.

[22] L.-H. Huang, H.-J. Hung, C.-C. Lin, and D.-N. Yang, "Scalable and bandwidth-efficient multicast for software-defined networks," in IEEE GLOBECOM'14, pp. 1890-1896.

[23] S.-H. Shen, L.-H. Huang, D.-N. Yang, and W.-T. Chen, "Reliable multicast routing for software-defined networks," in IEEE INFOCOM'15, 2015, pp. 181-189.

[24] P. Cameron, "Combinatorics: Topics, techniques, algorithms." Cambridge University Press, 1994.

[25] M. Fisher, G. Nemhauser, and L. Wolsey, "An analysis of approximations for maximizing submodular set functions-II," Springer Berlin Heidelberg, vol. 8, pp. 73-87, 1978.

[26] A. Caprara, H. Kellerer, U. Pferschy, and D. Pisinger, "Approximation algorithms for knapsack problems with cardinality constraints," European Journal of Operational Research, vol. 12, no. 2, pp. 333-345, 2000.

[27] "The internet topology zoo," 2014. [Online]. Available: http://www.topology-zoo.org/dataset.html

[28] D. Xu, M. Chiang, and J. Rexford, "Link-state routing with hop-by-hop forwarding can achieve optimal traffic engineering," IEEE/ACM Trans. on Networking, vol. 19, no. 6, pp. 1717-1730, Dec 2011. 
[29] "IBM ILOG CPLEX," 2014. [Online]. Available: http://www-01.ibm.com/software/commerce/optimization/cplex-optimizer/ 
(a) VtIWavenet2011

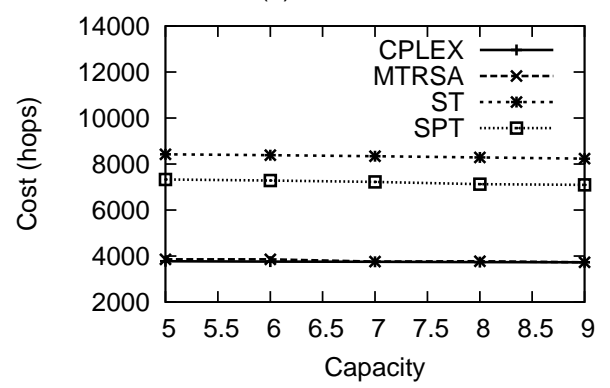

(b) Columbus

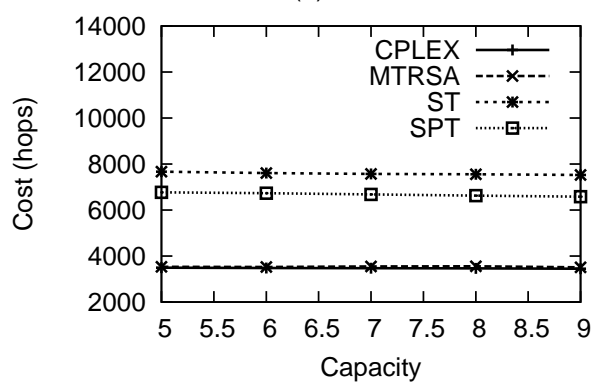


(a) VtIWavenet2011

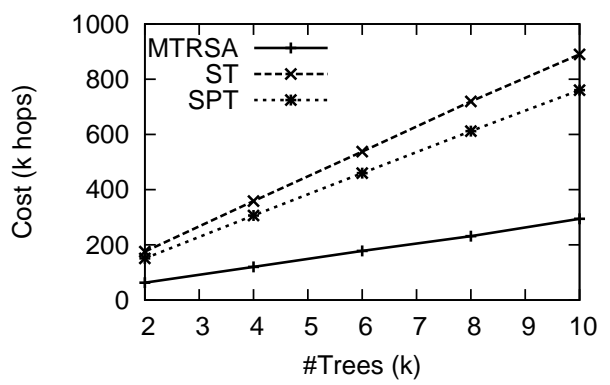

(b) Columbus

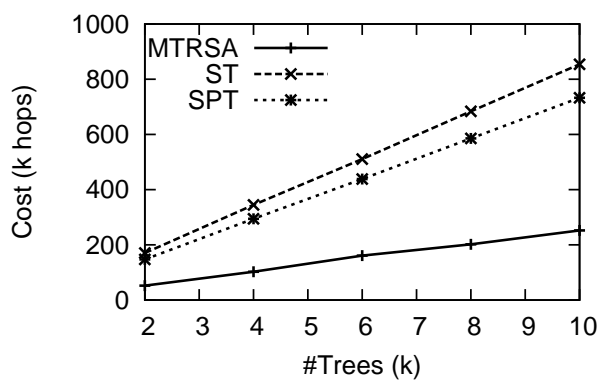


(a) VtIWavenet2011

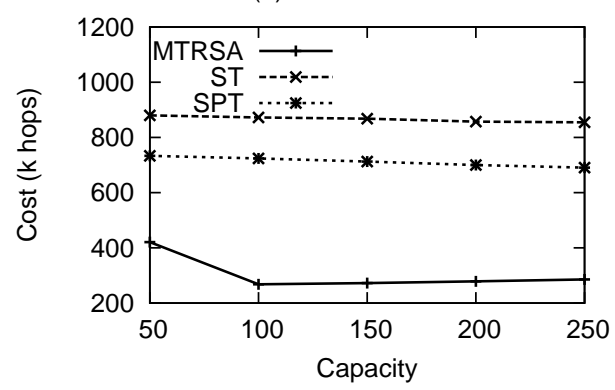

(b) Columbus

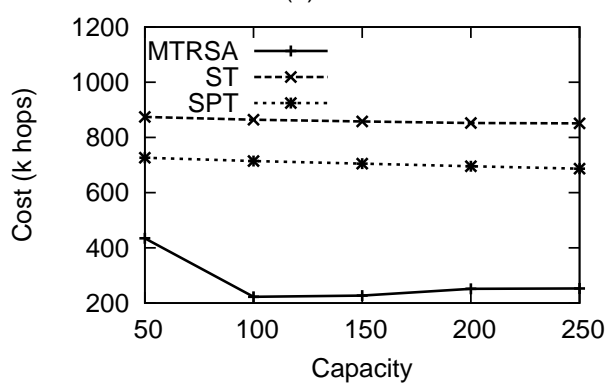


(a) VtIWavenet2011

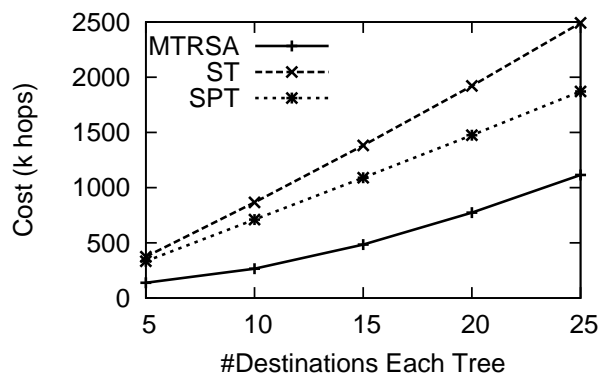

(b) Columbus

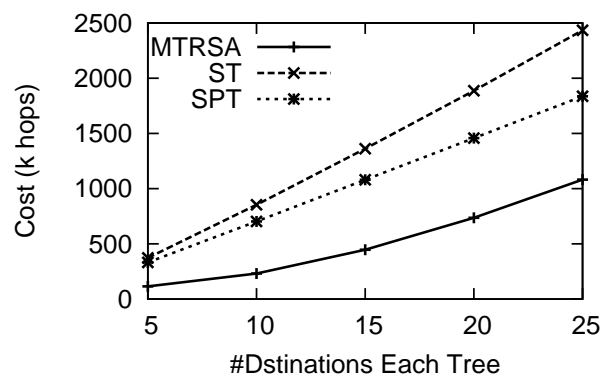


(a) VtIWavenet2011

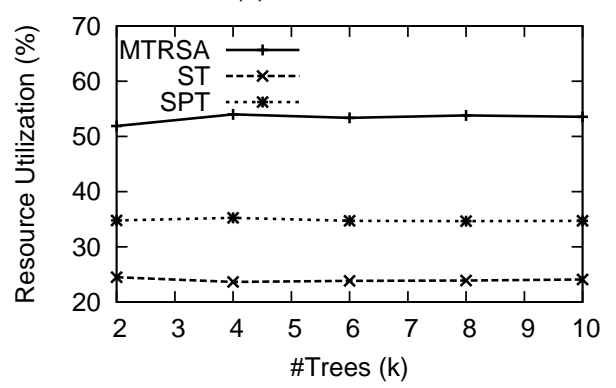

(b) Columbus

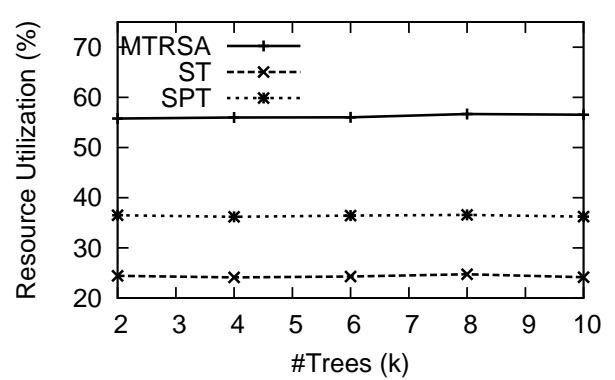


(a) VtIWavenet2011

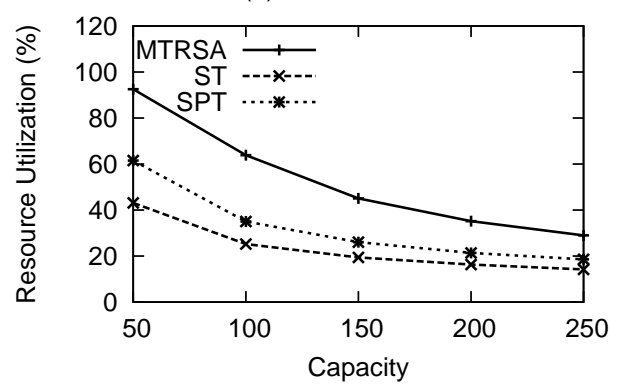

(b) Columbus

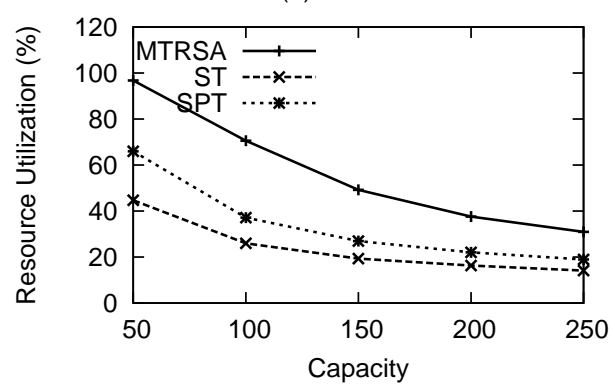


(a) VtIWavenet2011

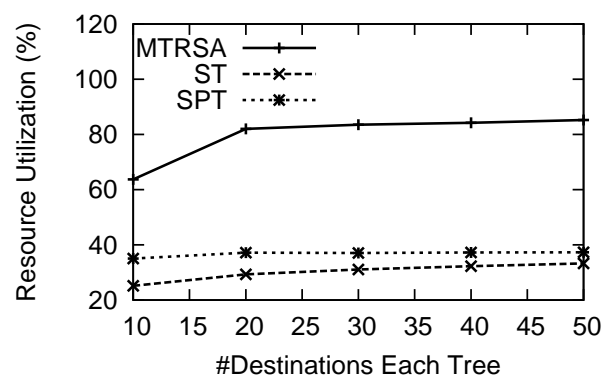

(b) Columbus

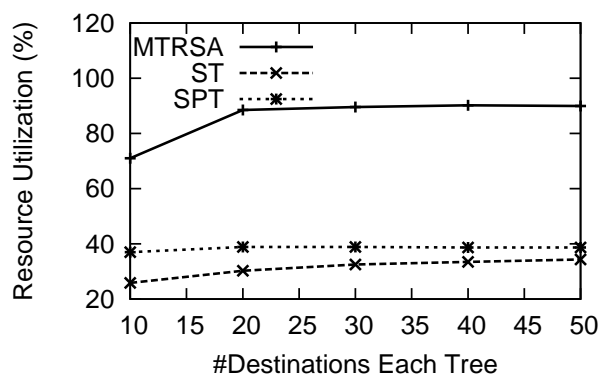

